\title{
TWO CASES OF THORACIC ACTINOMYCOSIS IN CHILDREN
}

BY

\author{
FREDA M. PAUL \\ From the Paediatric Unit, General Hospital, Singapore
}

(RECEIVED FOR PUBLICATION SEPTEMBER 7, 1962)

Most textbooks of paediatrics consider actinomycosis a rare infection in children and few collective studies or case reports in children have been reported. Pyogenic infection of the chest is common in Malaya and Singapore, but there has been no recorded case of actinomycosis in children so far, and it is because of the rarity of the condition that it is felt worth while to report these two cases seen during the past two years.

\section{Case Reports}

Case 1. An 8-year-old Chinese girl was admitted to the Paediatric Unit on March 10, 1960, because of pain in the left lower chest for two months and a swelling over the posterior aspect of the left lower chest for five days. The character of the pain, which was pricking in nature and aggravated by breathing, suggested that it was pleuritic in origin. The child was reported to be losing weight over the last two months.

The patient lived with her parents and three sisters on an island off Singapore, which was mainly a fishing area. The parents remarked that the neighbours reared cattle, but the child had not been in contact with these animals. She, however, had the habit of chewing grass occasionally.

Clinically, the child was febrile with a temperature of $100^{\circ} \mathrm{F}$. $\left(37 \cdot 8^{\circ} \mathrm{C}\right.$.) and underweight for her age (34 lb. $(15.4 \mathrm{~kg})$.$) . The main findings were in the chest which$ showed a swelling posteriorly over the lower aspect of the left side of the chest (Fig. 1). The trachea was central, and the physical findings were those of an effusion below the inferior angle of the scapula in the form of a dull percussion note, with diminished air entry and no adventitious sounds.

Our clinical diagnosis was that of an empyema necessitans and the following investigations were done.

The haemoglobin was $9 \mathrm{~g} . / 100 \mathrm{ml}$., the anaemia being largely due to ankylostomiasis. The total white count was $21,600 /$ c.mm. with polymorphonuclears $70 \%$, lymphocytes $18 \%$ and monocytes $6 \%$. The Mantoux test $1 / 1,000$ and $1 / 100$ was negative and the erythrocyte sedimentation rate was $97 \mathrm{~mm}$. (Westergren). A chest radiograph showed an opacity in the left lower base and, on screening, the pleural opacity in the left base was postero-lateral in location. The swelling over the chest wall corresponded to the area of pleural density suggesting continuity between the two. No bony lesion of the ribs was detected.

A diagnostic tap of the chest was done, and three drops of thick pus were obtained, which on microscopic examination showed filaments of actinomycosis. Culture of the sputum grew Actinomycosis bovis and a pure growth of Actinobacillus actinomycetemcomitans and Streptococcus viridans.

The child was given injections of crystalline penicillin 200,000 units six hourly for one month and then maintained on phenoxymethyl penicillin (Oracyn) tablets as an out patient for another month.

Clinically the air entry was good over the left lung two months later. A repeat radiograph of the chest two months later showed clearing of the consolidation of the left base. Throat swab and sputum were cultured from members of the family and the immediate neighbours, but none of them showed evidence of actinomycosis on culture.

Case 2. A 6-year-old Chinese boy was admitted on May 22, 1961, to the Paediatric Unit because of chronic discharging sinuses over the anterior aspect of the chest for over a year. The parents applied Chinese medicine over the sinuses, which had made the lesions worse, and the sinuses were discharging yellow pus for a month before admission.

The child was also noted to have a cough for three months, productive of yellow sputum. There was a mild fever which did not worry the parents much and for five days before admission the patient was breathless.

On examination, the general condition of the child was good in spite of his long illness. He was not breathless, but the striking feature was the presence of large sinuses over the anterior aspect of the chest, which were discharging thick yellow pus (Fig. 2).

The trachea was central and on percussion there was dullness over the right chest below the inferior angle of the scapula and the right axilla, and coarse crepitations were heard over these areas. The liver was enlarged three finger breadths and it was very firm.

A radiograph of the chest on May 22, 1961, revealed a diffuse opacity occupying the whole of the right chest 
(Fig. 3). No fluid or pus was obtained on aspiration. Repeated cultures of the sputum for actinomycosis and for tuberculosis were negative. The child was therefore treated with sulphatriad and chloramphenicol intramuscularly for 14 days in the ward and then with oral penicillin tablets.

A repeat radiograph of the chest in October 1961 (Fig. 4a, b) showed some clearing of the consolidation of the right upper and middle lobes, but there was an erosion of the fifth right rib anteriorly.

The provisional diagnosis was actinomycosis, but initially repeated examination of the sputum microscopically and on culture showed no evidence of actinomycosis or tuberculosis.

The haemoglobin was $6 \mathrm{~g} . / 100 \mathrm{ml}$., the anaemia being largely due to ankylostomiasis. The white cell count was $12,000 / \mathrm{c} . \mathrm{mm}$. with polymorphonuclears $62 \%$, lymphocytes $28 \%$, eosinophils $6 \%$ and monocytes $6 \%$.

The pus showed Gram positive diplococci and on culture Pneumococcus was grown. The Mantoux 1/1,000 and $1 / 100$ was negative.

A biopsy of the sinuses on May 30, 1961, showed evidence of subacute inflammation with heavy deposits of haemosiderin but no fungi were seen on section.

The secondary infection from the sinuses except for one sinus healed with persistent antibiotic therapy. A repeat biopsy of this sinus on May 15, 1962, showed several colonies of fungi with the morphological aspects of actinomycosis.

The child improved with antibiotic therapy over the year and gained $5 \mathrm{lb}$. (2.26 kg.); the sinuses were healing and the chest was better except for an area of dullness posteriorly, which had diminished air entry. The liver which had been enlarged three finger breadths was now not palpable. The sputum grew $A$. israeli on two occasions. The child had some dental caries and $A$. israeli were grown from the extracted teeth.

This child is being followed up closely as there is a possibility that the lesions may recur. The home conditions were carefully investigated with the help of our almoner, particularly the physical environment in the hope that it would have some bearing on our search for the source of fungi. The family live in a very old attap house on the outskirts of a hillock in Singapore. The immediate surroundings were haphazardly planted with rubber trees. The ground in front of the patient's house was full of rotting undergrowth.

Inside the house there were sheets of latex hung over the beams of the bed. The home conditions appeared ideal for the growth of any fungus, but so far our search has not been successful. The other members of the family were not affected with actinomycosis.

\section{Discussion and Comments}

Human actinomycosis is widespread all over the world and juvenile actinomycosis has been reported in England, France, Norway, Italy, Japan and America. However, childhood actinomycosis is rare.

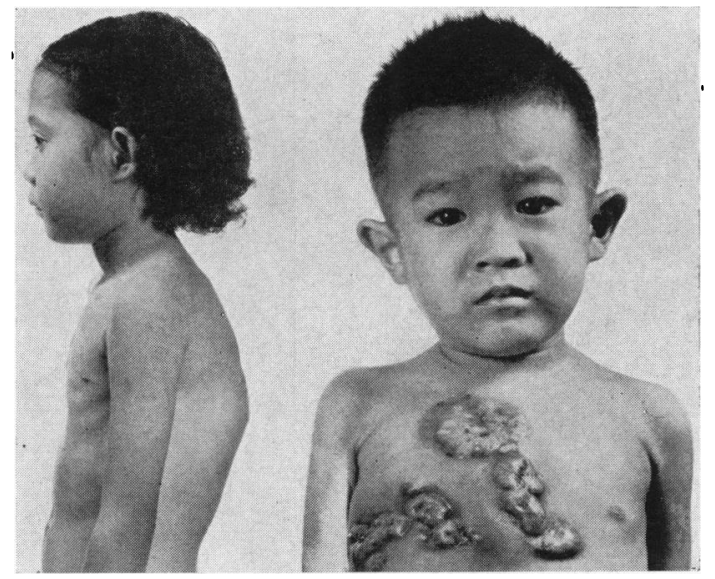

Fig. 1.

Fig. 2.

Fig. 1.-Case 1. Note the swelling in the lateral view of the chest. Fig. 2.-Case 2 with the chronic discharging sinuses over the anterior aspect of the chest.

In Sanford's and Voelker's survey of 670 cases in the United States in 1925 they were able to find only 45 cases in children, 15 years old or younger. In recent years there have been few recorded cases.

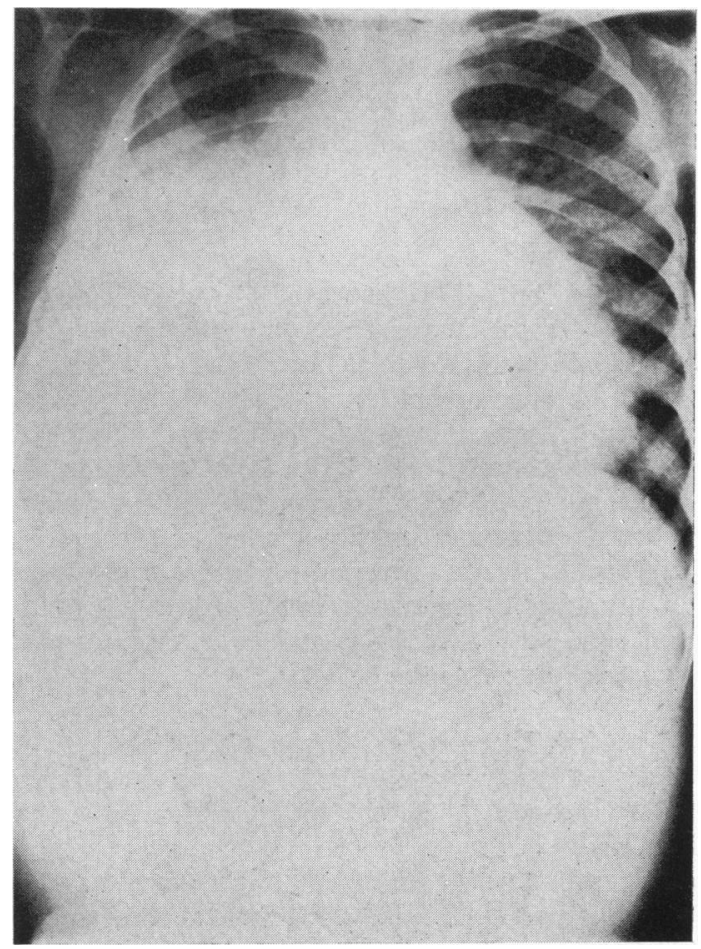

Fig. 3.-Case 2. Opacity occupying the whole right chest. 

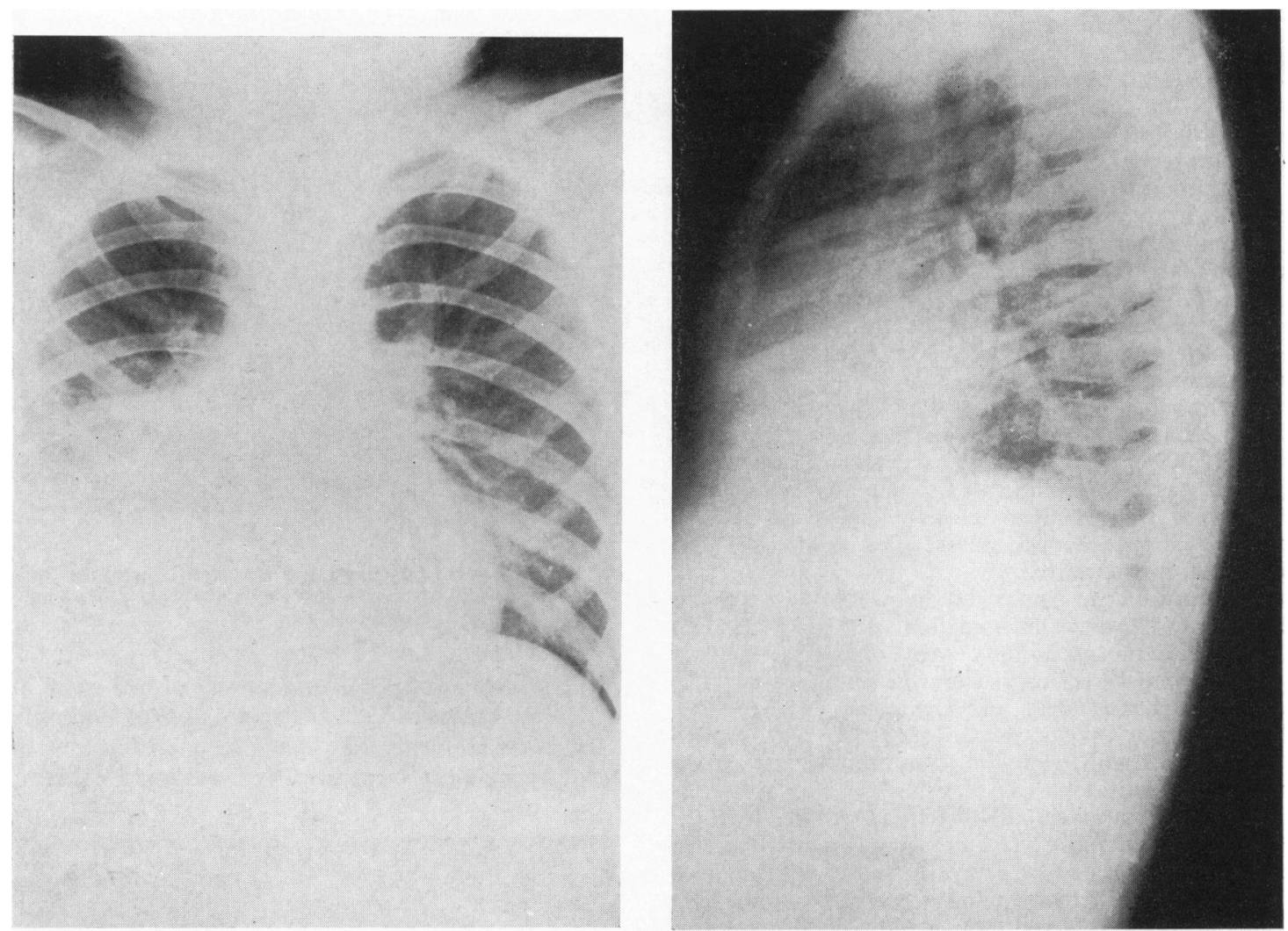

FIG. 4a and b.-Radiographs of the chest one year later show clearing of the opacity on the right side, with some deformity of the fifth right rib anteriorly.

Figi and Cutts (1931) reported 14 cases in children among 450 patients with actinomycosis seen by them. The cervico-facial area was involved in 10 and the abdomen in four cases. An outstanding point of difference in the histories of children and adults was the absence of previous trauma in the former group. They also reported that a history of dental extractions or penetration of the oral or pharyngeal mucous membrane with grasses, which so commonly precedes the onset of the disease in adults and so forms the portal of entry, was conspicuously absent in the children.

Types of Thoracic Actinomycosis. Cope (1938) has divided thoracic actinomycosis into three types, namely bronchial, parenchymal and mediastinal actinomycosis.

The first type is rare with slight symptoms of bronchitis and actinomyces is found in the sputum. Parenchymal disease begins generally in the lower lobes with involvement later of the rest of the lung. The lymph nodes and lymphatics are seldom involved; the pleura shows little resistance and the presence of an effusion becomes purulent and progression to empyema formation is noticed. This was so in Case 1. The mediastinal type involves the thoracic wall and leads to abscess formation. The bodies of the thoracic vertebrae are involved, and pleural effusion and empyema are also early features.

Jepson, Rose and Tonkin (1958) reported a case in an adult who presented with pain in the interscapular region and with a cold abscess over the neck. The abscess on aspiration showed goldenyellow pus with sulphur granules.

Bacteriology. It is now generally established that $A$. israeli is not the same species as $A$. bovis or cattle actinomycosis or the species, usually aerobic, found 
in grasses and soil (Wright, 1905; Cope, 1938; Weed and Baggenstoss, 1949).

In 1877 Bollinger published an account of the condition and from that time onwards the label $A$. bovis is attached to the particular type found in the cow by Bollinger. This strain is anaerobic. The second type, which is a smaller group, is also anaerobic and is known as $A$. israeli. $A$. israeli has been found in teeth and infected tonsils of normal people (Naeslund, 1925; Davis, 1941 ; Slack, 1942). It probably requires trauma or locally reduced resistance with an associated synergic aerobe to establish a lesion.

Treatment. The formation of the actinomycotic granule also has some bearing on treatment, for a whole colony of actinomycosis is apt to exhibit greater in vitro resistance to a particular chemotherapeutic agent than does a suspension of the organism.

If the patient is to be permanently cured, drug therapy must be intense enough to suppress all organisms, including those harboured within the protective confines of actinomycotic granules. Failure to penetrate these densely clumped colonies has undoubtedly accounted for many instances of recurrent infection. In earlier years therapeutic efficiency in treatment of actinomycosis has been ascribed to a number of agents, i.e. iodides, thymol, copper sulphate and autogenous vaccine. Clemens (1940) reported recovery in a child with actinomycosis after thymol therapy.

The potential value of penicillin in actinomycosis was first cited in 1941 by the Oxford investigators (Abraham, Chain, Fletcher, Gardner, Heatley, Jennings and Florey). It has been found that the organism was killed by a concentration of 0.01 units of penicillin per ml. of medium.

Over the succeeding years it has become obvious that higher doses of penicillin would be required, especially in disseminated infections and in severe types of pulmonary and abdominal actinomycosis.

Peabody and Seabury (1960) state that for the average case of actinomycosis, penicillin remains the drug of choice. It must be given in moderately high dosage and for sustained periods of time. They use 1 to 6 million units of penicillin daily in adults for a minimum of six to eight weeks. For severe cases, they state that 12 million units per day is not excessive. Nevertheless, Jepson et al. (1958) advocate the combination of penicillin and streptomycin. Apart from the synergistic effect of streptomycin with penicillin, the streptomycin is useful against the other organisms that are nearly always present.

Associated organisms like Actinobacillus actinomycetemcomitans, and other Gram negative bacilli which may be present, may be resistant to penicillin. It is generally agreed that these organisms are responsible for initiating actinomycotic lesions but what the exact role is in perpetuating infections is not known.

The treatment of actinomycosis is akin to tuberculosis in that it must be prolonged for many months and sometimes for over a year, not only to eradicate all signs of the disease but to preclude the possibility of reactivation.

Generally speaking, the broad spectrum antibiotics do show greater in vitro effectiveness against $A$. bovis than does sulphadiazine. Even so the prolonged administration of a broad spectrum antibiotic has certain inherent hazards and for this reason their routine use either alone or in combination is probably unwise.

Regardless of such claims, Peabody and Seabury (1960) say that penicillin still remains the undisputed drug of choice.

\section{Summary}

Two cases of actinomycotic infection of the chest in children are described.

The incidence, bacteriology and treatment are discussed.

I wish to thank Dr. M. Adams and Dr. J. Stein for their bacteriological assistance, and Dr. Quah Quee Guan and Professor Wong Hock Boon, Paediatric Unit, General Hospital, Singapore for their helpful criticisms. My thanks are also due to the Director of Medical Services for permission to publish this article.

\section{REFERENCES}

Abraham, E. P., Chain, E., Fletcher, C. M., Gardner, A. D., Heatley, N. G., Jennings, M. A. and Florey, H. W. (1941). Further observations on penicillin. Lancet, 2, 177.

Bollinger, $O$. (1877). Quoted by Cope, Z. (1938).

Cope, Z. (1938). Actinomycosis. Oxford University Press, London. Clemens, H. H. (1940). Actinomycosis; report of a case in a child

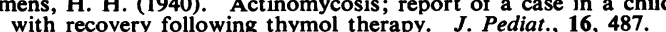

with recovery following thymol therapy. J. Pediat., 16, 487 .
Davis, M. I. J. (1941). Analysis of forty-six cases of actinomycosis with reference to its etiology. Amer. J. Surg., 52, 447.

Figi, F. A. and Cutts, R. E. (1931). Actinomycosis in childhood. Amer. J. Dis. Child., 42, 279.

Jepson, E. M., Rose, F. C. and Tonkin, R. D. (1958). Thoracic actinomycosis. Brit. med. J., 1, 1025.

Naeslund, C. (1925). Studies of actinomyces from the oral cavity. Acta path. microbiol. scand., $2,110$.

Peabody. J. W. and Seabury, J. H., Jr. (1960). Actinomycosis and nocardiosis. Amer. J.Med., 28, 99.

Sanford, A. H. and Voelker, M. (1925). Actinomycosis in the United States. Arch. Surg., 11, 809.

Slack, J. (1942). The source of infection in actinomycosis. J. Bact., 43, 193.

Weed, L. A. and Baggenstoss, A. J. (1949). Actinomycosis. A pathologic and bacteriologic study of 21 fatal cases. Amer. J. clin. Path., 19, 201.

Wright, J. H. (1905). The biology of the microorganism of actinomycosis. J. med. Res., 13, 349. 